УДК 519.873

\title{
Methods of Assessing the Characteristics of the Multiprocessor Computer System Adaptation Unit
}

\author{
Sergey N. Efimov* \\ Informatics and Telecommunications Institute \\ Reshetnev Siberian State Aerospace University \\ Krasnoyarskii Rabochii, 31, Krasnoyarsk, 660037 \\ Russia \\ Valeriy N. Tyapkin ${ }^{\dagger}$ \\ Dmitry D. Dmitriev $\ddagger$ \\ Military Engineering Institute \\ Siberian Federal University \\ Svobodny, 79, Krasnoyarsk, 660041 \\ Vitaly A. Terskov ${ }^{\S}$ \\ Informatics and Telecommunications Institute \\ Reshetnev Siberian State Aerospace University \\ Krasnoyarskii Rabochii, 31, Krasnoyarsk, 660037 \\ Russia
}

Received 05.02.2016, received in revised form 31.03.2016, accepted 02.05.2016

The article presents the results of the development of mathematical models of the main methods for assessing efficiency indicators of multiprocessor computer systems adaptation unit multibeam antenna in real time and task of selecting the optimal structure.

Keywords: mathematical modeling, multiprocessor computer system, performance, reliability. DOI: 10.17516/1997-1397-2016-9-3-288-295.

\section{Introduction}

Currently, a growing number of satellite communication systems developers and manufacturers pay attention to the adaptive multibeam antenna systems. These systems allow you to implement flexible traffic management by forming a multi-beam patterns within the service area. In addition, they have such an important property, as the high noise immunity when exposed as a deliberate and industrial noise provided by the use of spatial filtering methods for interference.

The adaptive multibeam antenna can be built on the basis of a phased antenna arrays with scanning beams and multibeam antennas are constructed on the basis of hybrid reflector antennas [1]. The main control element of such antennas is a block adaptation that implements control algorithms and individual beams beamforming. A block adaptation is a processor based on the adaptive signal processor, and the adaptation is made on the program level. In [2] made

\footnotetext{
*efimov@bk.ru

†tyapkin58@mail.ru

$\ddagger$ dmitriev121074@mail.ru

\$terskovVA@mail.ru

(c) Siberian Federal University. All rights reserved
} 
the development of synthesis algorithms of the amplitude-phase distribution of hybrid reflector antenna, which must be implemented in the adaptive processor. The complexity of these algorithms require the use of multi-processor computer systems (MCS) [3].

In the works of many researchers as key indicators by which assesses the effectiveness of the functioning of MCS, performance and reliability are taken [4-6]. In [7] were constructed analytical and simulation models, allowing to carry out an assessment of the main indicators.

\section{Development of mathematical models of evaluation of performance and reliability}

To estimate the performance operation of MCS process is represented by a closed system and with the expectation a random distribution of all types of queries on all bus bars with no interaction between them. It is assumed that the total flow of requests from each processor type is the simplest option to $v_{i}(i=1,2, \ldots, N)$, where $N$ is number of types of processors), and the service time submits exponential distribution with parameter $\mu_{i}$. Streams parameters depend on the values of $T_{0 i}$ - the relative operation time processor $i$-type. These dependencies are complex. In particular, if the processor is running at a higher relative speed, it does not follow that the requests to memory, it generates a greater intensity, as the higher performance can be explained by the greater complexity of the implemented features, in which the processor becomes almost self-contained and very rarely turns to shared memory.

Each processor in some random times require service. Service is carried out by memory access by bus $n$. New incoming service request is sent with equal probability in any of the tires and available for service is received. If bus are busy, incoming service request is queued and waiting to be of service.

The discipline of service is a random equiprobable choice of the queue. The system can be in the following states:

$a_{0,0, \ldots, 0}^{0,0}-$ system service requests not, available bus;

$a_{1,0, \ldots, 0}^{1,0}-$ system is one type of request from the first processor, in a single bus request is serviced, no queue;

$a_{0,0, \ldots, 1}^{1,0}-$ system is a request from a processor of $N$-type, one served by a bus request queue not;

$a_{j_{1}, j_{2}, \ldots, j_{N}}^{k, l}$-system is $j_{i}$ requests from processor $i$-type, where $i=1,2, \ldots, N, k$ bus busy service, $l$ are requests for service queues;

$a_{m_{1}, m_{2}, \ldots, m_{N}}^{h, M-h}-$ system is $m_{i}$ requests for each type processors, h bus busy service, $(M-h)$ are requests for service lines, where $M$ is total number of processors;

$$
\begin{gathered}
M=\sum_{i=1}^{N} m_{i}, \\
h= \begin{cases}n, & M>n, \\
M, & M \leqslant n ;\end{cases}
\end{gathered}
$$

$k=0,1, \ldots h ; l=0,1, \ldots,(M-k)$. 
The equations for stationary mode system is as follows [8]:

$$
\begin{aligned}
& -\sum_{i=1}^{N} m_{i} v_{i} P_{0,0, \ldots, 0}^{0,0}+\mu_{1} P_{1,0, \ldots, 0}^{1,0}+\mu_{2} P_{0,1, \ldots, 0}^{1,0}+\ldots+\mu_{N} P_{0,0, \ldots, 1}^{1,0}=0, \\
& {\left[-\sum_{i=1}^{N}\left(m_{i}-j_{i}\right) v_{i}+d_{i} \mu_{i}\right] P_{j_{1}, j_{2}, \ldots, j_{N}}^{k, l}+\left(m_{1}-j_{1}+1\right) v_{1} \frac{n-k+1}{n} P_{j_{1}-1, j_{2}, \ldots, j_{N}}^{k-1, l}+\ldots} \\
& \cdots+\left(m_{N}-j_{N}+1\right) v_{N} \frac{n-k+1}{n} P_{j_{1}-1, j_{2}, \ldots, j_{N}-1}^{k-1, l}+\left(m_{1}-j_{1}+1\right) v_{1} \frac{k}{n} P_{j_{1}-1, j_{2}, \ldots, j_{N}}^{k, l-1}+\ldots \\
& \cdots+\left(m_{N}-j_{N}+1\right) v_{N} \frac{k}{n} P_{j_{1}, j_{2}, \ldots, j_{N}-1}^{k, l-1}+\left[1-\left(\frac{k-1}{n}\right)^{l+1}\right] d_{1} \mu_{1} P_{j_{1}+1, j_{2}, \ldots, j_{N}}^{k, l+1}+\ldots \\
& \cdots+\left[1-\left(\frac{k-1}{n}\right)^{l+1}\right] d_{N} \mu_{N} P_{j_{1}, j_{2}, \ldots, j_{N}+1}^{k, l+1}+\left(\frac{k}{n+1}\right)^{l} d_{1} \mu_{1} P_{j_{1}+1, j_{2}, \ldots, j_{N}}^{k+1, l}+\ldots \\
& \cdots+\left(\frac{k}{n+1}\right)^{l} d_{N} \mu_{N} P_{j_{1}, j_{2}, \ldots, j_{N}+1}^{k+1}=0 \\
& -\sum_{i=1}^{N} \mu_{i} P_{m_{1}, m_{2}, \ldots, m_{N}}^{h, M-h}+v_{1} P_{m_{1}-1, m_{2}, \ldots, m_{N}}^{h, M-h-1}+v_{2} P_{m_{1}, m_{2}-1, \ldots, m_{N}}^{h, M-h-1}+\ldots+v_{N} P_{m_{1}, m_{2}, \ldots, m_{N}-1}^{h, M-h-1}=0,
\end{aligned}
$$

The solution of the resulting system of equations (3), taking into account the normalization condition

$$
P_{0,0, \ldots, 0}^{0,0}+\sum_{k=1}^{h} \sum_{l=0}^{M-k} \sum_{j_{1}=0}^{k+l} \sum_{j_{2}=0}^{k+l-j_{1}} \ldots \sum_{j_{N-1}=0}^{k+l-R} P_{j_{1}, j_{2}, \ldots, j_{N-1},(k+l-R)}^{k, l}=1
$$

to determine the probability $P_{j_{1}, j_{2}, \ldots, j_{N}}^{k, l}$ of finding the system in the states $a_{j_{1}, j_{2}, \ldots, j_{N}}^{k, l}[8]$ :

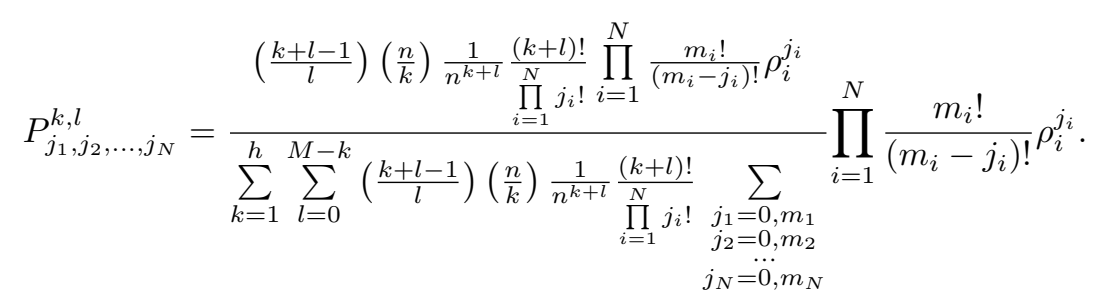

When evaluating MCS performance is determining the total number of requests in services and in queues, regardless of their type. The total number of requests in the queue can be determined using the concept of combined stationary probabilities $P_{k, l}$, the average queue length $m_{m i d}$ and coefficients $\theta_{i}$, relative losses of performance for each type of processor:

$$
\begin{gathered}
P_{k, l}=\sum_{j_{1}=0}^{k+l} \sum_{j_{2}=0}^{k+l-j_{1}} \cdots \sum_{j_{N}-1=0}^{k+l-R} P_{j_{1}, j_{2}, \ldots, j_{N-1},(k+l-R)}^{k, l}, \\
l=\sum_{k=1}^{h} \sum_{l=1}^{M-k} P_{k, l} \cdot l \\
\theta_{i}=1+\frac{l_{\mathrm{cp}} \tau_{i}}{T_{0 i}+\tau_{i}} .
\end{gathered}
$$


The relative performance of MCS may be determined using following equation

$$
\operatorname{Per}_{\text {mid }}=\sum_{i=1}^{N} k_{i} \frac{m_{i}}{\theta_{i}}
$$

where $k_{i}$ is coefficient taking into account the performance of each type of processor:

$$
k_{i}=\frac{T_{0 \min }}{T_{0 i}},
$$

where $T_{0 i}$ is the relative time execution of the operation processor $i$-type; $T_{0 \text { min }}$ is smallest value of $T_{0 i}$. The parameters $T_{0 \text { min }}$ and $T_{0 i}$ assumed to be constant and set before the process of performance evaluation.

Consider the approach to determining the reliability of the example of the MCS with an arbitrary number of processors and buses of different types. The process of functioning of MCS is seen as a succession of states in a certain time interval $\delta t$. This process can be described using the apparatus of queuing theory.

Consider MCS consisting of $N$ different types of processors by $m_{i}(i=1,2, \ldots, N)$ of each type of processors and shared memory. Combining processor shared memory according carried by $n$ buses, and in an extreme case the number of buses can be equal to the number of processors (full interface).

The total amount of memory units required is determined by its volume. An important parameter of the process is the coefficient the connection according to the defined not only the structure of the program being executed, but the special processors and speed, and the speed of memory-processor exchange, ie parameters $T_{0 \text { min }}$ and $T_{0 i}$.

It is expected that the total flow of failures of all types buses and processors submits Poisson distribution law with parameter $v_{i}$, and the recovery buses and processors $i$-type change exponential law with parameter $\mu_{i}$, and these parameters are determined by the speed of processors.

If a new incoming request to restore the service finds the device free, then it is taken to the service. If a query finds service are busy, it is queued and waiting to be of service. The discipline of service is a random equally possible choice of the queue. The system under consideration can be in the following states:

$a_{0,0,0, \ldots, 0}-$ interface and all processors are faulty and restored. The computational process is stopped.

$a_{1,0,0, \ldots, 0}-$ is working one bus interface, and $(\mathrm{m}-1)$ faulty bus and recovering. All processors are faulty and restored. The computational process is stopped.

$a_{0,1,0, \ldots, 0}-$ all interface bus are faulty and restored. All processors except for one of the first type are faulty and restored. The computational process is stopped.

$a_{0,0,1, \ldots, 0}-$ all interface bus are faulty and restored. All processors except for one of the second type, faulty and restored. The computational process is stopped.

$a_{0,0,0, \ldots, 1}-$ all interface bus are faulty and restored. All processors except for one N-type, faulty and restored. The computational process is stopped.

$a_{j_{1}, j_{2}, j_{3}, \ldots, j_{N-1}}-j_{1}$ bus interface operable and are involved in the computation process, and $\left(m_{1}-j_{1}\right)$ are faulty and restored; $j_{2}$ first type processors are serviceable and are involved in the computation process, a $\left(m_{2}-j_{2}\right)$ are faulty and restored; $j_{3}$ second type processors are serviceable and are involved in the computation process, and $\left(m_{3}-j_{3}\right)$ are faulty and restored; $j_{N-1}$ processor $N$-type are serviceable and are involved in the computation process, and $\left(m_{N-1}-j_{N-1}\right)$ are faulty and restored. 
$a_{m_{1}, m_{2}, m_{3}, \ldots, m_{N-1}}$ - all processors and bus included in the computer system, and are serviceable in a computing process involved.

We write the system of differential equations system [9]

$$
\begin{gathered}
\frac{d P_{0,0, \ldots, 0}(t)}{d t}= \\
=-\sum_{i=1}^{N+1} m_{i} \mu_{i} P_{0,0, \ldots, 0}(t)+\nu_{1} P_{1,0, \ldots, 0}(t)+\nu_{2} P_{0,1, \ldots, 0}(t)+\ldots+\nu_{N+1} P_{0,0, \ldots, 1}(t), \\
\frac{d P_{j_{1}, j_{2}, \ldots, j_{N+1}}(t)}{d t}=-\sum_{i=1}^{N+1}\left[\left(m_{i}-j_{i}\right) \mu_{i}+\nu_{i}\right] P_{j_{1}, j_{2}, \ldots, j_{N+1}}(t)+ \\
+\left(m_{1}-j_{1}+1\right) \mu_{1} P_{j_{1}-1, j_{2}, \ldots j_{N+1}}(t)+\left(m_{2}+j_{2}+1\right) \mu_{2} P_{j_{1}, j_{2}-1, \ldots, j_{N+1}}(t)+\ldots \\
\ldots+\left(m_{N+1}-j_{N+1}+1\right) \mu_{N+1} P_{j_{1}, j_{2}, \ldots, j_{N+1}-1}(t)+ \\
+\nu_{1} P_{j_{1}+1, j_{2}, \ldots, j_{N+1}}(t)+\nu_{2} P_{j_{1}, j_{2}+1, \ldots, j_{N+1}}(t)+\ldots+\nu_{N+1} P_{j_{1}, j_{2}, \ldots, j_{N+1}+1}(t), \\
\frac{d P_{m_{1}, m_{2}, \ldots, m_{N+1}}(t)}{d t}=-\sum_{i=1}^{N+1} \lambda_{i} P_{m_{1}, m_{2}, \ldots, m_{N+1}}(t)+\mu_{1} P_{m_{1}-1, m_{2}, \ldots, m_{N+1}}(t)+ \\
+\mu_{2} P_{m_{1}, m_{2}-1, \ldots, m_{N+1}}(t)+\ldots+\mu_{N+1} P_{m_{1}, m_{2}, \ldots, m_{N+1}-1}(t) .
\end{gathered}
$$

The number of states of the system is equal to $A=\prod_{i=1}^{N+1}\left(m_{i}+1\right)$.

The system of equations has a unique solution, taking into account the normalization condition. The analytical solution in a general form:

$$
P_{j_{1}, j_{2}, \ldots, j_{N}+1}=\frac{\frac{l !}{\prod_{i=1}^{N+1} j_{i} !} \prod_{i=1}^{N+1} \frac{m_{i} !}{\left(m_{i}-j_{i}\right) !} \rho_{i}^{j_{i}}}{\sum_{\substack{j_{1}=0, m_{1} \\ j_{2}=0, m_{2} \\ \ldots \ldots \ldots \ldots \ldots \\ j_{N}=0, m_{N+1} \ldots}} \frac{l !}{\prod_{i=1}^{N+1} j_{i} !} \prod_{i=1}^{N+1} \frac{m_{i} !}{\left(m_{i}-j_{i}\right) !} \rho_{i}^{j_{i}}} .
$$

The main indicators of reliability following MCS: $P^{\varepsilon}$ is probability of failure of a deliver the set performance; $k_{\Gamma}^{\varepsilon}$ is operating ratio with a given level of performance.

Given the probabilities of $P_{j_{1}, j_{2}, \ldots, j_{N+1}}$ a particular state of $a_{j_{1}, j_{2}, \ldots, j_{N+1}}$ the system MCS indicators of reliability are calculated as follows:

$$
\begin{gathered}
P^{\varepsilon}=\sum_{\substack{j_{1}=0, m_{1} \\
j_{2}=0, m_{2} \\
\ldots \ldots \ldots \ldots \ldots, \ldots \ldots, \ldots \\
j_{N+1}=0, m_{N+1}}} P_{j_{1}, j_{2}, \ldots, j_{N+1}}, \\
K^{\varepsilon}=\frac{P^{\varepsilon}}{P^{\varepsilon}-\left(1-P^{\varepsilon}\right)}=\frac{P^{\varepsilon}}{2 P^{\varepsilon}-1} .
\end{gathered}
$$

Constructed complex models can go to the formalization of tasks choice of effective variants of diverse MCS adaptation unit. The formalization of tasks choice of effective variants of the MCS should lead to optimization productions. In this group of three criteria are obvious:

- performance criteria which must be to maximize;

- the criteria of reliability, which should be maximized (readiness coefficients, time before failure, vitality, etc.) or minimized (probability of failure, while staying in an inoperable state); 
- the cost of the criteria which must be minimized (system cost, the cost of system development, maintenance costs, repair costs, etc.).

On the variables of this task will be imposed restrictions on energy consumption, speed, etc. Thus, the problem of choosing an effective option as a formalized MCS multicriteria optimization problem with three groups of conflicting criteria. When you select a leading criterion in each group get a problem with three criteria and a group of significant limitations, which in turn other criteria of each group.

A significant problem for the solution of the optimization problem also creates a method for calculating the objective functions (criteria). In [7] it has been proposed both analytical and simulation models. However, from the point of view of established algorithms for optimization problems both classes of models is extremely inconvenient. Analytical models cannot be studied using mathematical analysis because of their extreme complexity and their analytical form does not give them any advantage over simulations. Thus, the objective function of the optimization problem can be considered a given algorithmically and its solution can only be employed methods of direct search.

Consider the type of variable optimization problem. In this case, we assume given the maximum number of types of processors $N$ and bus $(n)$ and the value of $T_{0 i}$ - he relative operation time processor $i$-type, as well as the maximum and minimum possible number of processors and bus of each type (for processors $m_{i}^{+}$and $m_{i}^{-}$respectively, $i=1,2, \ldots, N$, a tire $n_{j}^{+}$and $n_{j}^{-}$ respectively, $j=1,2, \ldots, n)$.

Let $m_{i}$ processors $i$-type included in the structure of the MCS $(i=1,2, \ldots, N)$, and by $n_{j}-$ number of tires $j$-type included in the MCS. It can be seen that the variables of the optimization problem $\left(m_{i}\right.$ and $\left.n_{j}\right)$ are integral, ie, there is a problem of discrete optimization, or more precisely - the task of optimizing the integer lattice.

In the case of the use of analytical models to estimate performance indicators, a list of variables is exhausted it. However, this task variables actually depend on the parameters and the processor bus $\left(v_{i}, \mu_{i}\right)$, which must be known in advance.

Here is the formal record of the constructed model optimization MCS structure:

$\operatorname{Per}_{\text {lead }}\left(m_{1}\left(v_{i}, \mu_{i}\right), \ldots, m_{i}\left(v_{i}, \mu_{i}\right), \ldots, m_{N}\left(v_{i}, \mu_{i}\right), n_{1}\left(v_{1}, \mu_{1}\right), \ldots, n_{j}\left(v_{j}, \mu_{j}\right), \ldots, n_{n}\left(v_{n}, \mu_{n}\right)\right) \rightarrow \max$

$\operatorname{Rel}_{\text {lead }}\left(m_{1}\left(v_{i}, \mu_{i}\right), \ldots, m_{i}\left(v_{i}, \mu_{i}\right), \ldots, m_{N}\left(v_{i}, \mu_{i}\right), n_{1}\left(v_{1}, \mu_{1}\right), \ldots, n_{j}\left(v_{j}, \mu_{j}\right), \ldots, n_{n}\left(v_{n}, \mu_{n}\right)\right) \rightarrow \max ;$

$\operatorname{Cost}_{\text {lead }}\left(m_{1}\left(v_{i}, \mu_{i}\right), \ldots, m_{i}\left(v_{i}, \mu_{i}\right), \ldots, m_{N}\left(v_{i}, \mu_{i}\right), n_{1}\left(v_{1}, \mu_{1}\right), \ldots, n_{j}\left(v_{j}, \mu_{j}\right), \ldots, n_{n}\left(v_{n}, \mu_{n}\right)\right) \rightarrow$ min $;$ under conditions

$\operatorname{Per}_{l}\left(m_{1}\left(v_{i}, \mu_{i}\right), \ldots, m_{i}\left(v_{i}, \mu_{i}\right), \ldots, m_{N}\left(v_{N}, \mu_{N}\right), n_{1}\left(v_{1}, \mu_{1}\right), \ldots, n_{j}\left(v_{i}, \mu_{i}\right), \ldots, n_{n}\left(v_{n}, \mu_{n}\right)\right) \geqslant$

$\geqslant \operatorname{Per}_{l}^{0}, l=1,2, \ldots, L_{P e r}$

$\operatorname{Rel}_{l}\left(m_{1}\left(v_{i}, \mu_{i}\right), \ldots, m_{i}\left(v_{i}, \mu_{i}\right), \ldots, m_{N}\left(v_{N}, \mu_{N}\right), n_{1}\left(v_{1}, \mu_{1}\right), \ldots, n_{j}\left(v_{i}, \mu_{i}\right), \ldots, n_{n}\left(v_{n}, \mu_{n}\right)\right) \geqslant$

$\geqslant \operatorname{Rel}_{l}^{0}, l=1,2, \ldots, L_{\text {Rel }}$;

$\operatorname{Cost}_{l}\left(m_{1}\left(v_{i}, \mu_{i}\right), \ldots, m_{i}\left(v_{i}, \mu_{i}\right), \ldots, m_{N}\left(v_{N}, \mu_{N}\right), n_{1}\left(v_{1}, \mu_{1}\right), \ldots, n_{j}\left(v_{i}, \mu_{i}\right), \ldots, n_{n}\left(v_{n}, \mu_{n}\right)\right) \leqslant$ $\leqslant \operatorname{SCost}_{l}^{0}, l=1,2, \ldots, L_{\text {Cost }}$

$m_{i}^{-}\left(v_{i}, \mu_{i}\right) \leqslant m_{i}\left(v_{i}, \mu_{i}\right) \leqslant m_{i}^{+}\left(v_{i}, \mu_{i}\right), i=1,2, \ldots, N, n_{j}^{-}\left(v_{j}, \mu_{j}\right) \leqslant n_{j}\left(v_{j}, \mu_{j}\right) \leqslant n_{j}^{+}\left(v_{j}, \mu_{j}\right)$, $j=1,2, \ldots, n$.

The following notation is used in this model:

$P e r_{l e a d}$ - leading performance evaluation criteria,

Rel lead - leading reliability evaluation criteria,

Cost $_{\text {lead }}$ - leading cost evaluation criteria,

$\operatorname{Per}_{l}, l=1,2, \ldots, L_{P e r},-$ secondary criteria for performance evaluation,

$\operatorname{Rel}_{l}, l=1,2, \ldots, L_{R e l},-$ secondary criteria for evaluating the reliability, 
$\operatorname{Cost}_{l}, l=1,2, \ldots, L_{\text {Cost }},-$ secondary criteria of valuation, $\operatorname{Per}_{l}^{0}, \operatorname{Rel}_{l}^{0}, \operatorname{Cost}_{l}^{0}$, - maximum permissible levels of secondary criteria.

The remaining symbols matching the above. We estimate the power of optimizing space. In real adaptation blocks multibeam antenna systems the number of types of processors can achieve twenty, and the number of each type of specialized processors can achieve several tens of (universal processors usually calculated ones). Assume that we have a special type of fifteen processors included in the system and possibly to 20 and each type of special processors to five purpose processors. Then, using an analytical model, we will have $5 \cdot 20^{15}$ possible combinations (without regard to whether any of them are valid on substantive limitations). Such is the power of space optimization. Significant restrictions will not significantly reduce the number of search points. This means that only a truncated sorting heuristic algorithms may be used to solve this problem.

Let's go back to the choice of effective performance specialty processors. In [7] it is shown that the performance of MCS depends on the number of special processors and their performance is nonlinear. This means that with an increase in speed of processors MCS productivity increases at the beginning and then starts to decrease due to conflicts when accessing memory. So you cannot rely on special processors maximum speed, and it is necessary to choose the design of optimal MCS to provide the best performance of all the MCS in general. For a formal statement of the problem, this means that the relative value operation time processor $i$-type $\left(T_{0 i}\right)$ may not be constant, but should also be included in the optimization of the number of variables. Moreover, they become "main" variables that determine the parameters of the system $\left(v_{i}, \mu_{i}\right)$, ie these parameters are functions of $T_{0 i}-\left(v_{i}\left(T_{0 i}\right), \mu_{i}\left(T_{0 i}\right)\right)$. This leads to considerable complication of the optimization problem, turning it into a two-level hierarchical problem:

$$
\left(\operatorname{Per}^{*}\left(T_{01}, \ldots, T_{0 i}, T_{0 N}\right), \operatorname{Rel}^{*}\left(T_{01}, \ldots, T_{0 i}, T_{0 N}\right), \operatorname{Cost}^{*}\left(T_{01}, \ldots, T_{0 i}, T_{0 N}\right)\right) \rightarrow e x t r,
$$

where $\operatorname{Per}^{*}, \operatorname{Rel}^{*}$ and $\operatorname{Cost}^{*}$ is solution of the problem of optimization of structure at MCS $v_{i}=v_{i}\left(T_{01}, \ldots, T_{0 i}, T_{0 N}\right), \mu_{i}=\mu_{i}\left(T_{01}, \ldots, T_{0 i}, T_{0 N}\right)$.

Variable upper hierarchy level task can be continuous (the problem of optimal design of structures) or discrete (the task of choosing an effective variant of the structure).

\section{Conclusion}

The proposed model for assessing the effectiveness and constructed on the basis of their formal model of select configurations allow specialized MCS go on to develop a decision support system for automated design of such systems and, subsequently, highly reliable and productive units adaptation multibeam antenna systems.

The research was financed by the Ministry of Education and Science of the Russian Federation (Agreement no. 14.577.21.0155, unique project RFMEFI57714X0155).

\section{References}

[1] V.N.Tyapkin, D.D.Dmitriev, T.G.Moshkina, Adaptive multibeam antennas, Vestnik of SibGAU, 42(2012), no. 2, 106-109 (in Russian). 
[2] V.N.Tyapkin, I.N.Kartsan, D.D.Dmitriev, A.E. Goncharov, Spatial Filtering Algorithms in Adaptive Multi-Beam Hybrid Reflector Antennas, 2015 International Siberian Conference on Control and Communications (SIBCON), 2015.

[3] M.B.Rutzig, A.C.Beck, L.S.Carro, Adaptive and dynamic reconfigurable multiprocessor system to improve software productivity, IET Computers and Digital Techniques, 9(2015), $63-72$.

[4] F.Bistouni, M.Jahanshahi, Reliability analysis of multilayer multistage interconnection networks, Telecommunication Systems, 2015.

[5] Sizhao Li, Shan Lin, Deming Chen, W. Eric Wong, Donghui Guo, Analysis of system reliability for cache coherence scheme in multi-processor, Eighth International Conference on Software Security and Reliability, SERE 2014, San Francisco, CA, USA, 2014, 247-251.

[6] W.Quan, A.D.Pimentel, Scenario-based run-time adaptive MPSoC systems, Journal of Systems Architecture, 62(2016), 12-23.

[7] S.N.Efimov, V.A.Terskov, Reconfigurable computing systems data processing and control, Informatsionnye tehnologii, Krasn. Inst. Zhel. Transp., Krasnoyarsk, 2013 (in Russian).

[8] V.A.Terskov, S.N.Efimov, Analytical method for estimating performance indicators for parallel computing systems, Informatsionnye tehnologii, 7(2003), 13-20 (in Russian).

[9] S.N.Efimov, Evaluation of reliability of distributed automated technological process control systems, Industrial Automatic Control Systems and Controllers, 9(2011), 9-13.

\section{Методы оценки характеристик многопроцессорного вычислительного комплекса блока адаптации}

Сергей Н. Ефимов

Институт информатики и телекоммуникаций Сибирский государственный аэрокосмический университет Красноярский рабочий, 31, Красноярск, 660037 Россия Валерий Н. Тяпкин Дмитрий Д. Дмитриев

Военно-инженерный институт Сибирский федеральный университет Академгородок, 13А, корпус 8, 660036

Россия

Виталий А. Терсков

Институт информатики и телекоммуникаций Сибирский государственный аэрокосмический университет Красноярский рабочий, 31, Красноярск, 660037

Россия

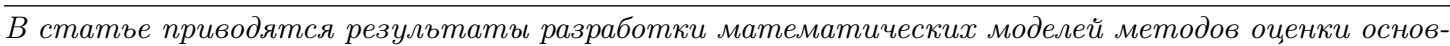
ных показателей эффективности многопроцессорных вычислительных комплексов блока адаптации многолучевой антенны в реальном времени и постановка задачи выбора их оптималъной структуры.

Ключевые слова: математическое моделирование, многопрочессорный вычислительный комплекс, производительность, надежность. 